Wright State University

CORE Scholar

$4-1-2008$

\title{
Effects of Annealing in N(2) Ambient on Traps and Persistent Conduction in Hydrothermally Grown ZnO
}

\author{
Z-Q. Fang \\ B. Claflin \\ David C. Look \\ Wright State University - Main Campus, david.look@wright.edu
}

Follow this and additional works at: https://corescholar.libraries.wright.edu/physics

Part of the Physics Commons

\section{Repository Citation}

Fang, Z., Claflin, B., \& Look, D. C. (2008). Effects of Annealing in N(2) Ambient on Traps and Persistent Conduction in Hydrothermally Grown ZnO. Journal of Applied Physics, 103 (7), 73714.

https://corescholar.libraries.wright.edu/physics/162

This Article is brought to you for free and open access by the Physics at CORE Scholar. It has been accepted for inclusion in Physics Faculty Publications by an authorized administrator of CORE Scholar. For more information, please contact library-corescholar@wright.edu. 


\title{
Effects of annealing in $\mathrm{N}_{2}$ ambient on traps and persistent conduction in hydrothermally grown $\mathrm{ZnO}$
}

\author{
Z.-Q. Fang, ${ }^{\text {a) }}$ B. Claflin, and D. C. Look \\ Semiconductor Research Center, Wright State University, Dayton, Ohio 45435, USA and Materials and \\ Manufacturing Directorate, Air Force Research Laboratory, Wright Patterson AFB, Ohio 45433, USA
}

(Received 21 December 2007; accepted 18 January 2008; published online 9 April 2008)

\begin{abstract}
Thermally stimulated current (TSC) spectroscopy and temperature-dependent dark current (DC) measurements have been applied to study traps and photoinduced persistent surface conduction in two hydrothermally grown bulk $\mathrm{ZnO}$ samples, as-grown, and annealed at $600{ }^{\circ} \mathrm{C}$ in $\mathrm{N}_{2}$ ambient for $30 \mathrm{~min}$, respectively. The as-grown sample had a room-temperature (RT) resistivity of 1.6 $\times 10^{3} \Omega \mathrm{cm}$, mobility of $2.1 \times 10^{2} \mathrm{~cm}^{2} / \mathrm{V} \mathrm{s}$, and carrier concentration of $1.8 \times 10^{13} \mathrm{~cm}^{-3}$, while the annealed sample was highly resistive, with RT resistivity of $3.6 \times 10^{6} \Omega \mathrm{cm}$, mobility of $4.4 \mathrm{~cm}^{2} / \mathrm{V} \mathrm{s}$, and carrier concentration of $3.9 \times 10^{11} \mathrm{~cm}^{-3}$. The as-grown sample showed strong conduction at low temperatures, which has been shown to be due to near-surface carriers in other studies. The annealed sample did not demonstrate this phenomenon. The dominant trap in the as-grown sample had an activation energy of $0.16 \mathrm{eV}$, was strongest near the surface, and is possibly related to $V_{\mathrm{Zn}}$. In the annealed sample, however, the dominant trap had an activation energy of $0.22 \mathrm{eV}$, was of bulk nature, and is tentatively assigned to $\mathrm{Li}_{\mathrm{Zn}}$. After several routine TSC measurements, the DC for the as-grown sample increased by more than one order of magnitude at low temperatures $(T<180 \mathrm{~K})$, while for the annealed sample, the DC increased by a factor of 2 at high temperatures $(T>200 \mathrm{~K})$. These effects are generated by the TSC trap-filling illumination and can persist for many days under vacuum. At RT, the DC in the annealed sample returns to its equilibrium state if the sample is vented to air. (C) 2008 American Institute of Physics.
\end{abstract}

[DOI: $10.1063 / 1.2903141]$

\section{INTRODUCTION}

Large $\mathrm{ZnO}$ crystals can presently be grown from the vapor phase (VP) ${ }^{1}$ melt (MLT), ${ }^{2}$ or water solution [hydrothermal (HYD)]. ${ }^{3}$ Unintentionally doped VP and MLT ZnO samples nearly always have low resistivities $(\sim 1 \Omega \mathrm{cm})$ due to high concentrations of uncompensated shallow donors. In contrast, HYD samples typically have much higher resistivities $(\sim 200-1000 \Omega \mathrm{cm})$ because the shallow donors are compensated by acceptors (e.g., $\mathrm{Li}$ ) present in the mineralizer, and the electrical properties are thus controlled by deep donors. In order to improve the quality of HYD-ZnO substrates for epitaxy, high-temperature annealings (e.g., $900{ }^{\circ} \mathrm{C}$ ) in $\mathrm{O}_{2}$, or $\mathrm{Ar}$ atmospheres have been investigated. In two different studies, ${ }^{4,5}$ it has been found that hightemperature annealing, in either $\mathrm{O}_{2}$ or Ar ambient, causes the substrate to convert from moderately resistive ( $n$ $\left.=10^{13}-10^{14} \mathrm{~cm}^{-3}\right)$ to conductive $\left(n=10^{15}-10^{16} \mathrm{~cm}^{-3}\right)$.

Deep centers in HYD-ZnO were first studied by Kuriyama et al., ${ }^{6}$ using thermally stimulated current (TSC) spectroscopy. Shortly thereafter, we used TSC to study deep centers in a HYD-ZnO sample [with room-temperature (RT) resistivity of $210 \Omega \mathrm{cm}$ ] irradiated with $1 \mathrm{MeV}$ electrons, and interestingly found that the sample became semiinsulating after irradiation. ${ }^{7}$ The dominant trap in the asgrown sample had an activation energy of $0.24 \mathrm{eV}$ and was tentatively assigned to $\mathrm{Li}_{\mathrm{Zn}}$ acceptors, while a new $0.15 \mathrm{eV}$ trap, possibly $V_{\mathrm{Zn}}$ related, was induced by the electron

${ }^{a)}$ Electronic mail: zhaoqiang.fang@wright.edu. irradiation. ${ }^{7}$ While carrying out TSC investigations of HYD$\mathrm{ZnO}$ samples, we have observed that the usual UV illumination, used to fill traps at $83 \mathrm{~K}$, also produces a subsequent increase of dark current (DC). We have evidence that this excess DC partially flows in the near-surface region, and will refer to this portion as photoinduced persistent surface conduction (PSC) rather than simply persistent photoconductivity, which is usually a bulk phenomenon. In the present study, we have annealed a HYD-ZnO sample at $600{ }^{\circ} \mathrm{C}$ in $\mathrm{N}_{2}$ ambient, which produced a change in resistivity from moderate to high, and we compare the changes in the TSC trap spectrum and the PSC.

\section{EXPERIMENTS}

Two, adjacent $5 \times 5 \times 0.5 \mathrm{~mm}^{3} \quad$ HYD-ZnO $c$-axis-oriented samples were cut from a larger 10 $\times 10 \mathrm{~mm}^{2}$ plate supplied by Tokyo Denpa. One sample (R59-2a) was used for control and the other (R-59-2c) was annealed in $\mathrm{N}_{2}$ ambient at $600{ }^{\circ} \mathrm{C}$ for $30 \mathrm{~min}$. Hall-effect measurements were performed at RT, using the van der Pauw method, with Ohmic contacts consisting of soldered indium dots on the corners. The RT electrical properties for the asgrown sample include resistivity of $1.6 \times 10^{3} \Omega \mathrm{cm}$, carrier concentration of $1.8 \times 10^{13} \mathrm{~cm}^{-3}$, and electron mobility of $2.1 \times 10^{2} \mathrm{~cm}^{2} / \mathrm{V} \mathrm{s}$.

TSC spectroscopy involves filling the electron and hole traps by illumination at low temperature and then warming the sample in the dark while recording the current produced by the thermal emission of carriers from the traps. To keep 


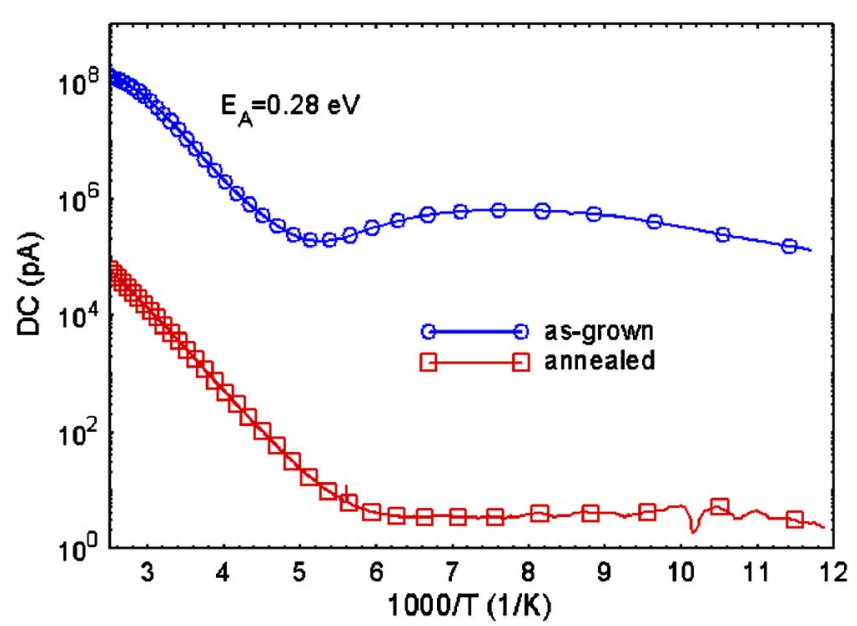

FIG. 1. (Color online) Arrhenius plot of DC, measured upon cooling at $V_{b}=1 \mathrm{~V}$ and $\beta=0.3 \mathrm{~K} / \mathrm{s}$, for as-grown and annealed HYD ZnO samples.

the initial conditions the same, the sample was always cooled from 400 to $83 \mathrm{~K}$ in the dark for each TSC scan. During cooling, the DC was measured as a function of temperature. The traps were then filled at $83 \mathrm{~K}$ with 360-nm (nearbandgap) illumination, produced by a $15 \mathrm{~W}$ halogen lamp and a bandpass filter. The photocurrent (PC) response was recorded at $83 \mathrm{~K}$ during a $5 \mathrm{~min}$ illumination period, and the decay of persistent PC (PPC) was recorded for a period of time ranging from $30 \mathrm{~s}$ to a few minutes after switching the light off. The TSC spectrum was then measured upon warming at a heating rate of $\beta=0.3 \mathrm{~K} / \mathrm{s}$ under a bias of $1 \mathrm{~V}$. From the TSC peak temperature $T_{m}$ for a given trap, an approximate activation energy can be determined from the relation ${ }^{8}$ $E_{T}=k T_{m} \ln \left(T_{m}{ }^{4} / \beta\right)$. A more accurate value of $E_{T}$ can be determined from an Arrhenius plot of $T_{m}^{4} / \beta$, where the heating rate $\beta$ typically varies from 0.1 to $0.4 \mathrm{~K} / \mathrm{s}$. Note that TSC characterization by itself cannot tell whether a peak is due to an electron trap or a hole trap. However, based on a comparison of the measured activation energies with those determined from samples previously studied by deep level transient spectroscopy or other means, we can speculate on the origins of the TSC traps observed in the as-grown and annealed samples. To demonstrate PSC, which can be produced by illumination with UV light at $83 \mathrm{~K}$, temperaturedependent DC data were compared before and after several TSC measurements.

\section{RESULTS AND DISCUSSION}

Arrhenius plots of the DC are presented in Fig. 1. From the figure, we see that (i) the DC at both high and low temperatures in the as-grown sample is more than three orders of magnitude higher than that in the annealed sample and (ii) both samples exhibit conductivity with an activation energy of about $0.28 \mathrm{eV}$ for $T>200 \mathrm{~K}$. In the as-grown sample, the high DC at low temperatures is evidently due to surface conduction, a conclusion which is supported by temperaturedependent Hall-effect (TDH) measurements on similar samples. Basically, the bulk carriers freeze out onto their parent donors at low temperatures and allow the more degenerate near-surface carriers to dominate the conduction. ${ }^{9,10}$

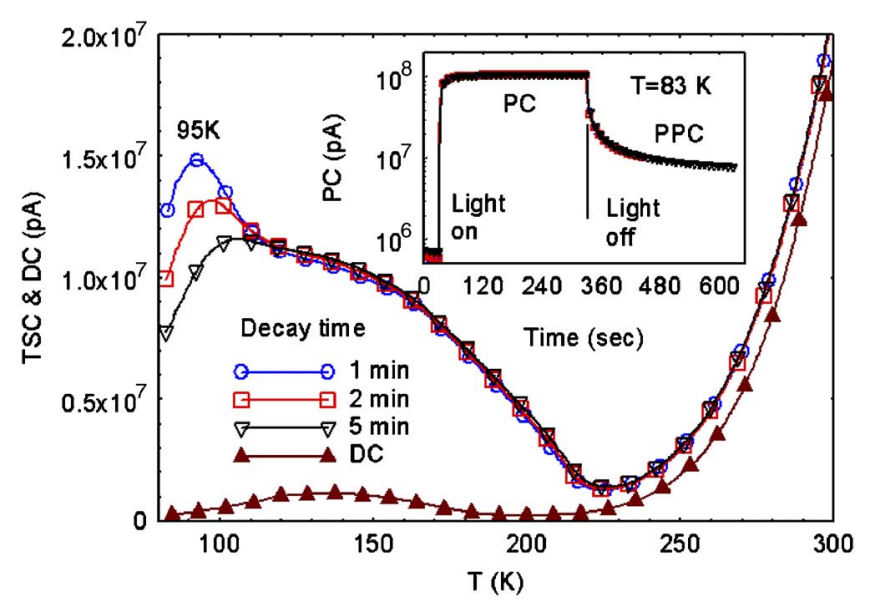

FIG. 2. (Color online) TSC spectra (360-nm illumination at $83 \mathrm{~K}$, sweep rate $\beta=0.3 \mathrm{~K} / \mathrm{s}$ ) and temperature-dependent $\mathrm{DC}$ measured at $V_{b}=1 \mathrm{~V}$ for the as-grown sample. Inset: PC responses at $83 \mathrm{~K}$ prior to each TSC sweep.

The $0.28 \mathrm{eV}$ center, similar to a $0.30 \mathrm{eV}$ center characterized by TDH measurements, ${ }^{11}$ is often found in HYD-ZnO samples and might be related to the oxygen vacancy $^{12}$ or impurities (Fe or $\mathrm{Ni}$ ). ${ }^{13}$ Figure 2 presents TSC and DC curves, along with $\mathrm{PC}$ responses, measured at $83 \mathrm{~K}$ for different decay times, shown in the inset. As mentioned earlier, a particular TSC sweep begins after the associated decay time $(1,2$, or $5 \mathrm{~min})$ is fulfilled. We find that each PC response consists of (i) an initial transient, which is related to a trap-filling process (ii) nearly saturated PC after 5 min illumination, which is associated with equal rates of thermal emission and captured by traps during photoexcitation, and (iii) a PPC decay after switching off the light, which in this case is mainly related to the thermal emission of carriers from shallow traps at $83 \mathrm{~K}$. In the TSC spectra, we clearly observe a $95-\mathrm{K}$ trap, which decreases in height as the decay time increases, owing to the increased thermal emission of carriers from that trap during the decay process. The 95-K trap, which has an estimated activation energy of $0.16 \mathrm{eV}$, is very similar to a $94-\mathrm{K}$ trap which is observed after electron irradiation $^{7}$ and, thus, probably associated with a point defect. From the value of activation energy, the most likely point defect is $V_{\mathrm{Zn}}$. At higher temperatures, the TSC and DC are of similar magnitudes and, thus, a subtraction of DC from TSC is necessary for a clear trap definition (called "net TSC spectrum"). Figure 3 presents TSC and DC for the annealed sample, along with the $83-\mathrm{K}$ PC responses which preceded each of the TSC sweeps. The annealed sample shows one order of magnitude lower PC and weaker PPC than the corresponding quantities found in the as-grown sample. In the TSC spectra of this sample, a dominant feature is observed at $135-\mathrm{K}$ with a small $95-\mathrm{K}$ shoulder on its rising edge. As expected for thermally emitted carriers, the amplitude of the $135-\mathrm{K}$ peak increases and the peak shifts to higher temperatures with increasing heating rate. From an Arrhenius analysis (not shown here), the activation energy of this dominant trap is $0.22 \mathrm{eV}$, which is similar to the energy expected for a $\mathrm{Li}_{\mathrm{Zn}}$ acceptor. ${ }^{7,14,15}$ It is difficult to directly compare the magnitudes of the TSC spectra of the as-grown and annealed samples because the TSC signal depends on the 


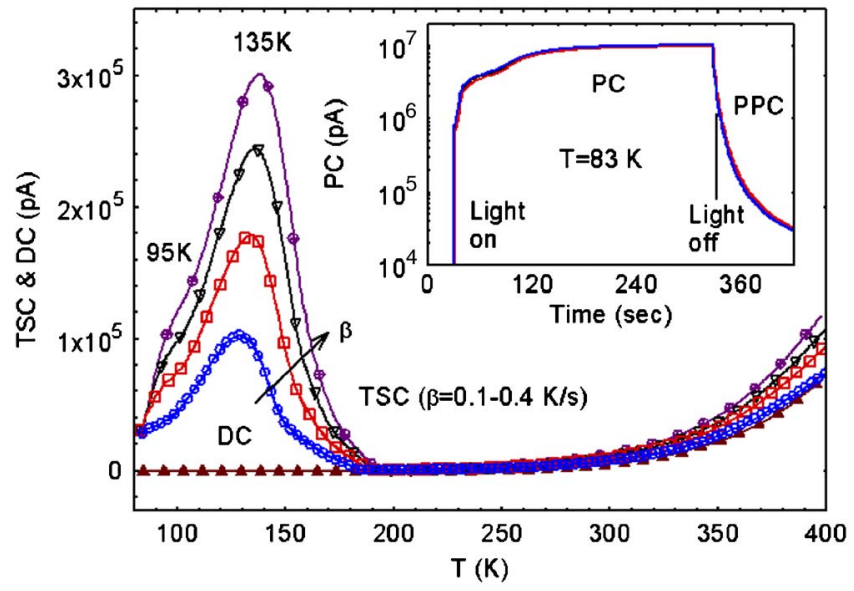

FIG. 3. (Color online) TSC spectra (360-nm illumination at $83 \mathrm{~K}$, sweep rate $\beta=0.1-0.4 \mathrm{~K} / \mathrm{s}$ ) and temperature-dependent DC measured at $V_{b}=1 \mathrm{~V}$ for the annealed sample. Inset: PC responses at $83 \mathrm{~K}$ (nearly identical) prior to each TSC sweep.

carrier lifetime in each case, and the carrier lifetimes are unknown. However, PC is also proportional to carrier lifetime, and thus, a rough normalization can be effected by dividing the net TSC signal by the PC signal. (Unfortunately, we only have $\mathrm{PC}$ at $83 \mathrm{~K}$ but the normalization should still be reasonably accurate for at least the low temperature traps.) Figure 4 shows the net, normalized TSC curves for the asgrown and annealed samples. From the figure, we see that, as compared to the annealed sample, the as-grown sample has a much higher TSC signal, with prominent 95, 135, and 160-K features, and two weak features at $275 \mathrm{~K}(0.56 \mathrm{eV})$ and $380 \mathrm{~K}(0.82 \mathrm{eV})$, which are close to those of two traps $(270$ and $375 \mathrm{~K}$ ) reported for an electron-irradiated HYD-ZnO sample. ${ }^{7}$ We speculate that the observation of high TSC signals in the as-grown sample is related to the traps existing in the surface region since it is known from $\mathrm{TDH}$ measurements that at least some impurities and defects, i.e., those producing shallow donors, are of much higher concentration near the surface than in the bulk. ${ }^{9,10}$ The annealed sample, on the other hand, does not have a strong surface conduction implying that at least some of the impurities and defects,

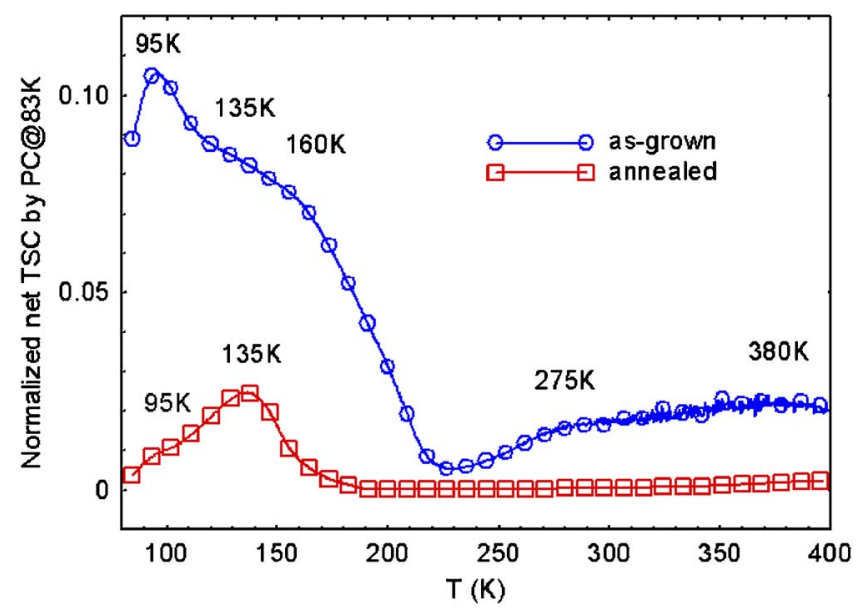

FIG. 4. (Color online) Normalized net TSC spectra (360-nm illumination at $83 \mathrm{~K}$, sweep rate $\beta=0.3 \mathrm{~K} / \mathrm{s}$ ) measured at $V_{b}=1 \mathrm{~V}$ for the as-grown and annealed samples.

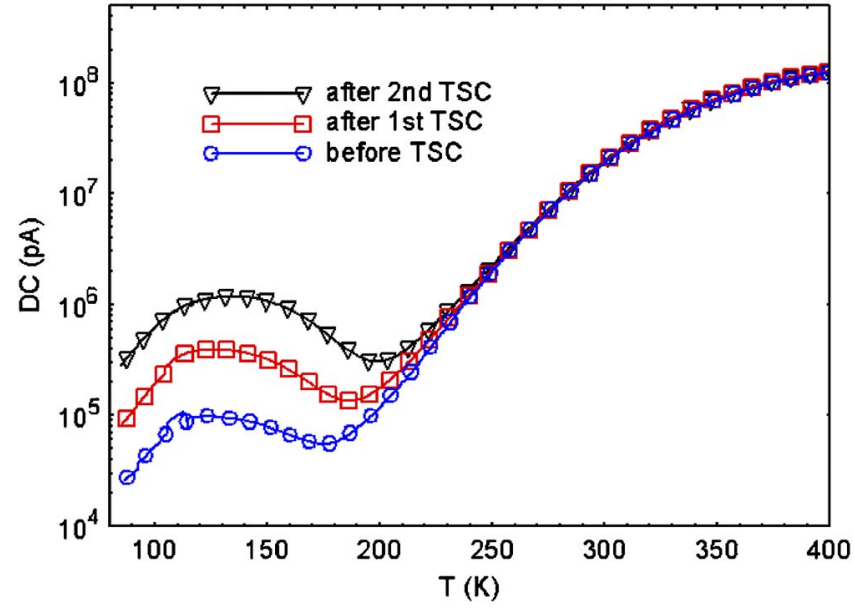

FIG. 5. (Color online) Temperature-dependent DC $\left(V_{b}=1 \mathrm{~V}\right.$ and $\beta$ $=0.3 \mathrm{~K} / \mathrm{s}$ ) during cooling scan, measured before and after several TSC measurements, for the as-grown sample.

perhaps those leading to traps, were removed by the anneal. If the anneal has indeed removed the shallow traps in the surface region, then the remaining shallow trap (at $135 \mathrm{~K}$ ) in the annealed sample is likely a bulk trap. An assignment of $\mathrm{Li}_{\mathrm{Zn}}$ for this trap would be consistent with the SIMS (secondary ion mass spectroscopy) observation of $\mathrm{Li}$ in the bulk of both annealed and unannealed samples. ${ }^{16}$

In the characterization of traps in these samples, we have observed that the DC continuously increases after each TSC measurement, especially for high resistivity or semiinsulating samples, such as electron-irradiated HYD-ZnO. ${ }^{7}$ (It should be remembered that each TSC measurement involves trap-filling light irradiation at the beginning of a temperature sweep.) Here, we present a similar observation for the HYD-ZnO samples used in this study, which were not irradiated. DCs, measured as usual during the cooling cycle before each set of TSC scans, are shown in Figs. 5 and 6 for the as-grown and annealed samples, respectively. For each sample, a large amount of time has been allowed to elapse before the first DC scan is recorded but the second and third DC scans occur after one or two TSC scans and, thus, after

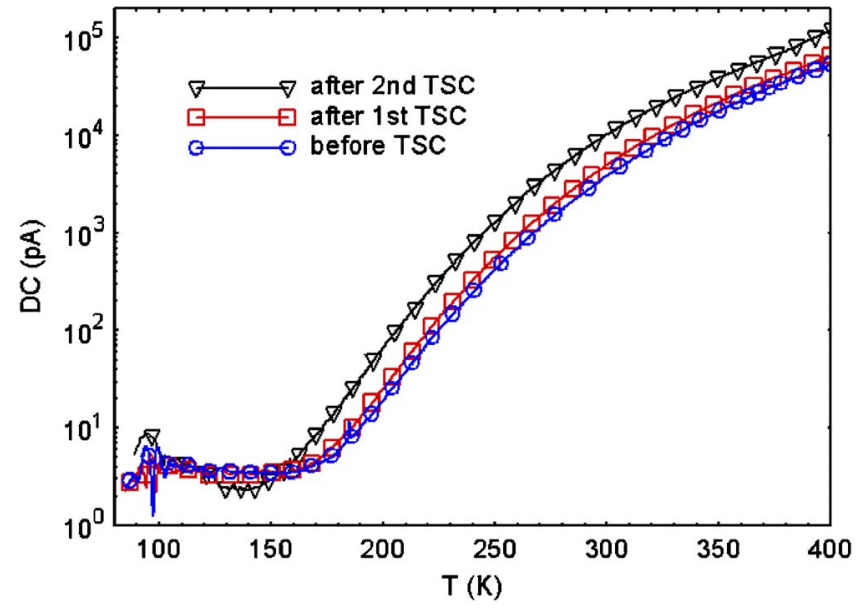

FIG. 6. (Color online) Temperature-dependent DC $\left(V_{b}=1 \mathrm{~V}\right.$ and $\beta$ $=0.3 \mathrm{~K} / \mathrm{s}$ ) during cooling scan, measured before and after several TSC measurements, for the annealed sample. 
the sample has been irradiated with light. After the second TSC scan, we see a significant increase of DC (more than one order of magnitude) in the as-grown sample but only at low temperatures (Fig. 5). For the annealed sample, on the other hand, the increase in DC is much smaller (about a factor of 2) and mainly occurs at higher temperatures (Fig. 6).

The strong DC temperature dependence at high temperatures in such samples evidently represents a simple carrierfreeze out process, ${ }^{9,10}$ involving a rather deep donor, about $0.3 \mathrm{eV}$. If so, then at equilibrium in the dark, the DC should vary as $\mathrm{DC} \propto n \propto\left(N_{D}{ }^{0} / N_{A}\right) \exp \left(-E_{D} / k T\right)$, where $N_{D}{ }^{0}$ and $N_{A}$ are the neutral-donor and total-acceptor concentrations, respectively, and $E_{D}$ is the donor activation energy. ${ }^{9,10}$ Here, we have assumed that $n \ll N_{D}, N_{A}$, that the acceptors are nearly all ionized $\left(N_{A}{ }^{-}=N_{A}-N_{A}{ }^{0} \approx N_{A}\right)$, and that the donors are partially ionized $\left(N_{D}{ }^{0}=N_{D}-N_{D}{ }^{+}=N_{D}-N_{A}{ }^{-}-n \approx N_{D}\right.$ $\left.-N_{A}\right)$. During and immediately after the application of the trap-filling light, the concept of an equilibrium Fermi level no longer holds because the traps themselves are not at equilibrium until they have all released their trapped carriers. We would have expected that the trapped carriers would all have been released by the end of each TSC sweep but Figs. 5 and 6 demonstrate that something has changed because the DC is higher after each TSC sweep has been completed. Since the DC curves after each successive TSC sweep are nearly parallel and continue to be dominated by the $0.3-\mathrm{eV}$ donor at the higher temperatures, it seems appropriate to model the system with a quasi-Fermi level that maintains a quasiequilibrium between the $0.3 \mathrm{eV}$ donor and the conduction band. That is, our representative equation now becomes DC* $\propto n^{*}$ $\propto\left(N_{D}{ }^{0 *} / N_{A}\right) \exp \left(-E_{D} / k T\right)$, where the asterisk denotes quasiequilibrium values. Such a quasiequilibrium state can be quite persistent, i.e., maintained for a long time in the dark, even at RT. There are at least two possible mechanisms for this effect.

1 The first mechanism involves the possibility of acceptorlike states at the $\mathrm{ZnO}$ surface, ${ }^{17}$ which in the dark will produce a depletion region and an associated electric field that drives electrons away from the surface and holes toward the surface. Thus, any electron-hole pairs created by the light in the depletion region, or within a diffusion length of the depletion region, will spatially separate, with the excess electrons dropping onto empty 0.3-eV donor states $\left(N_{D}{ }^{+}+e^{-} \rightarrow N_{D}{ }^{0}\right)$, and the excess holes residing at the surface. Because $N_{D}{ }^{0}$ is increasing to $N_{D}{ }^{0 *}, n$ will also increase to $n^{*}$, according to our formula. For an excess electron to recombine with an excess hole, the electron must have enough thermal energy to overcome a barrier $\left(0.3 \mathrm{eV}+V_{\mathrm{bi}}\right)$ where $V_{\mathrm{bi}}$ is the built-in voltage. Unfortunately, it is difficult to estimate $V_{\mathrm{bi}}$ because we do not have a detailed knowledge of $\mathrm{ZnO}$ surface states in the first place and also because the excess holes at the surface will compensate some of the negative charges there and reduce $V_{\mathrm{bi}}$. However, it is reasonable that a large-enough barrier could exist to maintain a persistent effect at RT.

2 A second mechanism involves photoexcitation of an electron (or multiple electrons) from a deep donor into the conduction band, followed by a reconfiguration of the lattice that moves the ionized state of the deep donor well into the conduction band, say, to an energy $E_{C}$ $+\Delta E$. Lany and Zunger ${ }^{18}$ have proposed such a mechanism for the oxygen vacancy $V_{\mathrm{O}}$, which in thermal equilibrium is deep (roughly $E_{C}-2 \mathrm{eV}$ ) and neutral. Their theoretical calculations find that if the two valence electrons of $V_{\mathrm{O}}$ are excited (in separate steps) to the conduction band (while the trap-filling light is on) the resultant state $V_{\mathrm{O}}^{++}+2 e^{-}$is pushed by the lattice reconfiguration into the conduction band to about $E_{C}+0.2 \mathrm{eV}$. The two available electrons would then normally be trapped by the resulting Coulomb potential into shallow, hydrogeniclike states near the conduction band. However, in our particular case, these two electrons will drop onto ionized (empty) donors, $0.3 \mathrm{eV}$ below the conduction band, thereby increasing $N_{D}{ }^{0}$ and, thus, also $n$. The increased $n$ is persistent, even at RT, because a thermal activation of about $0.5 \mathrm{eV}$ is required to return the $V_{\mathrm{O}}{ }^{++}+2 e^{-}$state to the deep $V_{\mathrm{O}}^{0}$ state.

In the annealed sample, the increased DC at $300 \mathrm{~K}$ is stable for many days in vacuum and for several hours in air. Recently, a conducting surface channel, associated with the sample ambient, was reported for Li-doped, high-resistivity, bulk $\mathrm{ZnO}$ samples by Schmidt et al. ${ }^{19}$ These authors found that under vacuum, a low-resistivity channel appears upon annealing up to $650 \mathrm{~K}$, while exposure to ambient air destroys the channel and returns the sample to a high-resistivity state. They claimed that the surface channel was only evident for samples showing high bulk resistivity and that it seemed to be the "natural" state of the $\mathrm{ZnO}$ surface. However, what we find is a photoinduced persistent current, which appears to be something different from the channel conduction reported in Ref. 19. That is, for both the as-grown and annealed samples, repeated DC measurements between 83 and $400 \mathrm{~K}$, prior to any TSC measurements, do not produce any significant changes in DC. Only after the 83-K trap-filling illumination does the DC increase. However, as was found in Ref. 19, exposure to air destroys the persistent current and leaves the annealed sample in a high resistivity state. This destruction of persistent current is consistent with the loss of UV-light induced persistent current in $p$-type $\mathrm{ZnO}$ after exposure to $\mathrm{O}_{2}$ gas. ${ }^{20}$ Again, if $V_{\mathrm{O}}$-related defects are responsible for our persistent currents, then exposure to $\mathrm{O}_{2}$ might be expected to decrease the effect.

\section{CONCLUSIONS}

In summary, TSC spectroscopy and temperaturedependent DC measurement have been used to investigate the traps and photoinduced persistent conduction in two HYD-ZnO samples, one as-grown and the other annealed in $\mathrm{N}_{2}$ ambient at $600{ }^{\circ} \mathrm{C}$ for $30 \mathrm{~min}$. The dominant trap at $95 \mathrm{~K}$ $(0.16 \mathrm{eV})$, observed in the as-grown sample, may be related to $V_{\mathrm{Zn}}$ defects in the surface region. The annealed sample exhibits much lower TSC signals with a dominant trap at $135 \mathrm{~K}(0.22 \mathrm{eV})$, which is tentatively assigned to $\mathrm{Li}_{\mathrm{Zn}}$ acceptors in the bulk. In the TSC studies, we find that illuminating 
the sample with UV light at $83 \mathrm{~K}$ not only optically fills traps but also produces a persistent DC, even after the traps have been totally emptied at the end of the TSC sweep. Part of this DC flows near the surface (PSC) and part of it flows in the bulk. After several consecutive TSC measurements, the DC for the as-grown sample increases by more than one order of magnitude at low temperatures $(T<180 \mathrm{~K})$, while the DC in the annealed sample increases by a factor of 2 at high temperatures $(T>200 \mathrm{~K})$. The increased DC at RT in the annealed sample can persist for days under vacuum but is destroyed by venting to air. Possible mechanisms for the persistent conduction have been discussed but further studies are needed to clarify its detailed nature.

\section{ACKNOWLEDGMENTS}

We wish to thank H. E. Smith, E. R. Heller, and G. C. Farlow for helpful discussions. Support was provided by NSF Grant No. DMR0513968 (L. Hess), ARO Grant No. W911NF-07-D-0001/Task07275 (M. Gerhold), AFOSR Grant No. FA9550-07-1-0013 (K. Reinhardt), and AFRL Contract No. FA8650-06-D-5401 (D. Silversmith).

${ }^{1}$ D. C. Look, D. C. Reynolds, J. R. Sizelove, R. L. Jones, C. W. Liton, G. Cantwell, and W. C. Harsch, Solid State Commun. 105, 399 (1998); ZN Technology, 910 Columbia Street, Brea, CA 92821.

${ }^{2}$ J. Nause and B. Nemeth, Semicond. Sci. Technol. 20, S45 (2005).
${ }^{3}$ K. Maeda, M. Sato, I. Niikura, and T. Fukuda, Semicond. Sci. Technol. 20, S49 (2005).

${ }^{4}$ S. Graubner, C. Neumann, N. Volbers, B. K. Meyer, J. Bläsing, and A. Krost, Appl. Phys. Lett. 90, 042103 (2007).

${ }^{5}$ G. H. Kassier, M. Hayes, F. D. Auret, M. Mamor, and K. Bouziane, J. Appl. Phys. 102, 014903 (2007).

${ }^{6}$ K. Kuriyama, M. Ooi, K. Matsumoto, and K. Kushida, Appl. Phys. Lett. 89, 242113 (2006)

${ }^{7}$ Z.-Q. Fang, B. Claflin, D. C. Look, and G. C. Farlow, J. Appl. Phys. 101, 086106 (2007).

${ }^{8}$ D. C. Look, Semicond. Semimetals 19, 75 (1983).

${ }^{9}$ D. C. Look, H. L. Mosbacker, Y. M. Strzhemechny, and L. J. Brillson, Superlattices Microstruct. 38, 406 (2005).

${ }^{10}$ D. C. Look, Surf. Sci. 601, 5315 (2007).

${ }^{11}$ D. C. Look, Mater. Sci. Eng., B 80, 383 (2001).

${ }^{12}$ J. C. Simpson and J. F. Cordaro, J. Appl. Phys. 63, 1781 (1988),

${ }^{13}$ Y. Jiang, N. C. Giles, and L. E. Halliburton, J. Appl. Phys. 101, 093706 (2007).

${ }^{14}$ O. Lopatiuk, L. Chernyak, A. Osinsky, and J. Q. Xie, Appl. Phys. Lett. 87, 214110 (2005).

${ }^{15}$ M. G. Wardle, J. P. Goss, and P. R. Briddon, Phys. Rev. B 71, 155205 (2005).

${ }^{16}$ H. E. Smith, personal communication.

${ }^{17}$ J. D. Prades, A. Cirera, J. M. Morante, and A. Cornet, Thin Solid Films 515, 8670 (2007).

${ }^{18}$ S. Lany and A. Zunger, Phys. Rev. B 72, 035215 (2005).

${ }^{19}$ O. Schmidt, A. Geis, P. Kiesel, C. G. Van de Walle, N. M. Johnson, A. Bakin, A. Waag, and G. H. Dohler, Superlattices Microstruct. 39, 8 (2006).

${ }^{20}$ B. Claflin, D. C. Look, and D. R. Norton, J. Electron. Mater. 36, 442 (2007). 